\title{
Stereotypes as Shared Beliefs: Effects of Group Identity on Dyadic Conversations
}

\author{
Minoru Karasawa \\ Nagoya University \\ Nobuko Asai and Yoshiko Tanabe \\ Kobe University
}

\begin{abstract}
Two experiments examined the influence of stereotypes on dyadic conversations. Undergraduate students listened to a recorded interview of a student who was described as either a member of their in-group or of an out-group. The interview contained stereotypeconsistent (SC) and stereotype-inconsistent (SI) descriptions of each group. Participants' conversations about the stimulus person were content-analyzed. One most consistent result was that for the out-group target, participants made more SI than SC utterances and spent more time discussing SI information. The difference between SC and SI utterances disappeared (Study 2) or was reversed (Study 1) for the in-group target. These patterns were observed particularly when the stimulus information contained a balanced combination of SC and SI characteristics. Furthermore, the stereotypicality of the conversations was related not only to the participant's own judgments but also to the partner's judgments. The significance of studying stereotypes as collectively shared intergroup attitudes is discussed.
\end{abstract}

KEYWORDS conversation, group identity, shared cognition, stereotype-consistent and inconsistent

STEREOTYPIC beliefs concerning social groups and categories often entail a high level of consensus in their contents. Likely sources of this concordance include social learning from parents, peers, and mass media. The collectively shared nature exemplifies the significance of stereotypes and prejudices not merely as products of intra-individual processes (e.g. effects of salience, categorization, and biased information processing) but also as phenomena at the intergroup level (Oakes, Haslam, \& Turner, 1994). In recent years, researchers from different perspectives, including the social cognition approach (e.g. Gardner, 1994; Stangor \& Schaller, 1996), the social identity perspective (e.g. Haslam et al., 1996), and communication research (e.g. Ruscher, 2001) have begun to pay attention to the status of stereotypes as collectively shared

\footnotetext{
$\overline{\text { Author's note }}$

Address correspondence to Minoru Karasawa, Department of Psychology, School of Informatics and Sciences, Nagoya University, Nagoya 464-8601, Japan [email: mkarasawa@nagoya-u.jp]
} 
reality (Hardin \& Higgins, 1996; Kashima, 2000; Lyons \& Kashima, 2003).

In the process of social learning of stereotypes, communication without doubt plays a critical role. For instance, conversations with family members and friends often contain stereotypic remarks which will in turn facilitate the formation and maintenance of collectively shared representations (e.g. Ruscher, 2001; Van Dijk, 1987). The main purpose of the present study was to investigate how stereotypic expectations influence conversations about a member of a group. Specifically, we focused on how people discuss stereotype-consistent (SC) and stereotypeinconsistent (SI) information in dyadic conversations. The significance of examining SC and SI information lies in the fact that these two kinds of information appear to be related, respectively, to the confirmation and disconfirmation of stereotypes. As such, examining the impact of SC and SI information in conversations was expected to reveal bases of the maintenance and possible changes of stereotypes as shared reality.

\section{Stereotypes and communication}

The consensual nature of stereotypes, and the communicative aspect in particular, has only recently regained researchers' attention. Consequently, little is known about how stereotypes are formed and used at the collective level in ongoing social processes. Among the relatively rare attempts to reveal such processes, the research program developed by Ruscher and her colleagues has provided important implications for the study of stereotype-based communication (e.g. Ruscher \& Duval, 1998; Ruscher \& Hammer, 1994; Ruscher, Hammer, \& Hammer, 1996). In their experimental paradigm, participants are invited in pairs and are presented with descriptions of traits and behaviors of a target individual including SC and SI cases. They are then asked to discuss their impressions of the target. The results of content analyses of these dyadic conversations typically demonstrated more SC than SI utterances, both in terms of the number of comments and their duration (see Ruscher, 1998, 2001).

The findings by Ruscher and her colleagues are important because they have demonstrated that dyadic conversations are likely to concentrate on SC parts of stimulus information of an individual group member. However, a number of qualifications should be noted. For instance, the predominance of SC conversations has been found typically in experimental settings where participants were first presented with behavioral information and later with the target category label (e.g. alcoholic) (e.g. Ruscher \& Hammer, 1994). This result may be interpreted, at least in part, by the fact that information processing tends to be susceptible to confirmatory bias especially in reconstruction situations (Snyder \& Uranowitz, 1978). Also, evidence shows that the memory for inconsistent information is more likely to deteriorate than the memory for consistent information (Dijksterhuis \& van Knippenberg, 1995). When the category label was given at the time of encoding, the concentration of conversations on SC information was found only when the dyads were under a need to reach consensus (Ruscher et al., 1996, Experiment 1). Furthermore, Ruscher et al. (1996, Experiment 2) found that when the dyads were led to believe that they had to make accurate judgments about the targets, they came to discuss more on SI instances rather than SC. Also, SI utterances increased when the dyad members needed to integrate divided information into a shared impression (Ruscher \& Duval, 1998). Taken together, evidence from a series of studies by Ruscher and her colleagues suggest that conversations can be more directed toward SI information when the dyads need to perform elaborate processing to form a coherent and accurate judgment.

In the present study, we employed a problemsolving situation to facilitate the participants' pursuit of accuracy. As we will describe in the Method section below, the participants were led to believe that there were 'correct' answers for the problems that they were required to discuss, purportedly on the basis of the stimulus person's actual responses. An anticipation of such veridical reality could give rise to an especially high level of need for accuracy compared to traditional manipulations such as introducing interpersonal dependency (Fiske \& Neuberg, 1990) and accountability (e.g. Ruscher et al., 1996). 
Some indirect support for this contention can be found in research evidence on group decision making. Members of a group tend to devote their discussion to shared information rather than to information that is unshared and uniquely held by different members (e.g. Stasser \& Titus, 1985). However, Stasser and Stewart (1992) found that this bias toward shared information can be reduced when the task given to the group pertains to problem-solving with an expectation for a true answer rather than reaching a consensual judgment. Parallel to this finding, we propose that a problem-solving task may also divert our participants from their conversations on SC characteristics of a target, which often serve as shared expectations, and rather lead them to discuss unexpected SI information.

Going back to the discussion on the research paradigm used by Ruscher and colleagues, another peculiar aspect is that their studies were almost exclusively concerned with conversations about a member of a stigmatized out-group (e.g. an alcoholic, a paraplegic, or a homosexual presented to college student participants). It appears important, however, to examine whether the observed effects apply particularly to an outgroup target or can be extended to any social groups including the perceiver's own in-group. It has been well established that communicative acts markedly vary depending on whether the exchanged information pertains to the in-group or the out-group (e.g. Harasty, 1997; Maass, 1999; Wigboldus, Semin, \& Spears, 2000). Likewise, the comparison between in-group and outgroup targets is expected to provide insight into potentially different information processing at the dyadic level involving the two kinds of targets. Discussions of such likely differences are in order.

\section{Effects of expectancy-consistent and inconsistent information}

From a broader perspective, the comparison between SC and SI information can be seen as a subclass of a general issue of expectancyconsistent versus inconsistent information. A large amount of evidence shows that information consistent with prior expectations tends to be overrepresented in our memory and judgments (e.g. Fiskl \& Taylor, 1991; Klein \& Snyder, 2003). However, inconsistent information is at times remembered better than consistent information and may exert a greater influence on judgments (e.g. Hastie \& Kumar, 1979; Rojahn \& Pettigrew, 1992; Stangor \& McMillan, 1992). One plausible explanation for this counterintuitive finding is that inconsistent information can be processed in a more elaborate way in order to be incorporated into a coherent target representation, and thus lead to a greater number of associative links (e.g. Srull \& Wyer, 1989). The recall advantage of inconsistent information has also been demonstrated with regard to stereotypes. For instance, Bardach and Park (1996) presented undergraduate participants with a number of statements describing either a male or female protagonist engaging in a masculine or feminine (i.e. SC or SI) behavior. The results showed that SI instances were retained to a greater extent than SC cases, particularly when the target was an out-group member (i.e. opposite-sex protagonist) rather than an in-group member (same-sex). Bardach and Park (1996) explained that the out-group target likely elicited more elaborate processing because of stronger stereotypic expectations.

The findings reviewed above are all concerned with information processing by an individual perceiver. It can be predicted, however, that the same effect may take place at the collective level when perceivers attempt to form shared representations. As long as perceivers, whether as individuals or in a group, are in need of attaining coherent understanding of a target, they may well show the tendency of elaborate processing for SI information. The present study examined whether such thorough processing might be reflected in conversations about an out-group member.

The above discussion should not be misinterpreted as pointing to an apparently related and yet distinctly different phenomenon called the Outgroup Homogeneity Effect (OHE). Past studies have established that the prevalence of SC instances among out-group members is often exaggerated and the frequency of SI instances 
tend to be underestimated, whereas the opposite is the case for in-group members (e.g. Linville, Salovey, \& Fischer, 1986; Park, Judd, \& Ryan, 1991). Caution is necessary because the OHE normally refers to a biased perception about a group as a whole, whereas the present discussion is focused on the representation of individual members. The OHE has been demonstrated in studies that employed group-level measurement of perceived homogeneity, such as estimates of frequency distributions concerning stereotype-relevant characteristics among group members, range estimates of these characteristics, and ratings of the overall similarity among group members (see Park \& Judd, 1990). However, the fact that the out group as a whole tends to be perceived in a more stereotypic (i.e. SC-based) manner does not necessarily mean that judgments about individual members are made only in an SC manner. Rather, evidence shows that judgments concerning individual out-group members are polarized. That is, SC characteristics of an out-group member are certainly more likely to be assimilated into the group-level stereotype, whereas SI characteristics can be even more contrasted away from the group stereotype and thus exaggerated (Linville \& Jones, 1980). Hence, both SC and SI information can call for attention as long as the target is an out-group individual. On the top of this baseline possibility of polarized attention, out-group members are likely to be subject to thorough examination on their SI characteristics, as we have pointed out earlier, because of potentially stronger stereotypic expectations for the outgroup than for the in-group.

\section{Consequences of conversations}

In addition to our main interest in the content of stereotype-related conversations, we also explored their subsequent influences on judgments about the target. According to previous studies, we may expect that people make judgments in a manner that corresponds to what they communicate (e.g. Higgins \& Rholes, 1978). To the extent that conversations are focused on SC (or SI) information, the impression of the target may lean toward a stereotypic direction (or vice versa) (e.g. Ruscher \& Duval, 1998). In the analysis of these relationships between dyadic conversations and judgments, a methodological caution is necessary regarding the potential interdependence of the dyadic data. That is, because of conversations, scores from participants within dyads are likely to be similar to each other on any index. Consequently, the similarity can be greater within rather than between dyads, and the potential redundancy can lead to inflated estimates of correlations and sample sizes if we simply calculate correlations with all of the individual participants as the unit of analysis (Griffin \& Gonzalez, 1995). To rule out the possibility of such biased estimates, we employed a method developed by Gonzalez and Griffin (1997) illustrated in Figure 1. Our primary interest was in examining the correlation between an individual participant's stereotypic utterances about the target (Variable A) and her stereotypic ratings (Variable B) as well as the correlation found for the conversation partner's utterances (A') and ratings (B'). As noted earlier, however, the dyadic conversation is expected to lead to within-dyad similarity with regard to the utterances $\left(r_{\mathrm{aa}}\right)$ and the ratings $\left(r_{\mathrm{bb}}\right)$. The Gonzalez-Griffin method allows us to test the statistical significance of correlations estimated for each individual (i.e. $r_{\mathrm{ab}}$ and $r_{\mathrm{a}{ }^{\prime} \text { ' }}$, separately), after controlling for the potential interdependence within the dyad. (Because the correlation is estimated for the same individual, we call this a 'self-correlation'.) Another advantage of this method is that we can also examine the association between variables across the dyad members (i.e. $r_{\mathrm{ab}}, r_{\mathrm{a}^{\mathrm{b}}}$ ), which we call a 'cross-correlation'. ${ }^{1}$ In our data, this refers to the unique correlation between stereotypic comments by one individual and impression ratings made by the conversation partner, again after controlling for the other potential correlations within the dyad. The analysis of cross-correlations has special significance for the purpose of our study because this method allows us to examine the potential relationship between conversations and impressions that are shared at the dyadic level. 


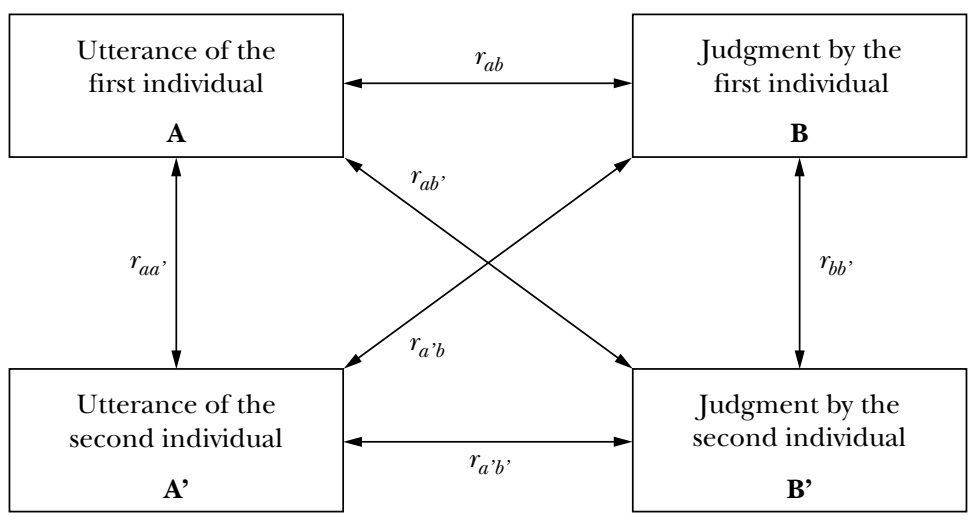

Figure 1. A schematic representation of the analysis of cross interclass correlations.

Note: A and B represent the analyzed variables, while A vs. A' and B vs. B' respectively distinguish the dyadic partners.

\section{Study 1}

Previous studies of stereotypes and communication have been mainly concerned with judgments about an out-group as the target (e.g. Kashima, 2000; Ruscher, 1998). It is not entirely clear whether the effects are limited to the out-group target or generalized to the in-group as well. To clarify this point, we manipulated the target's group identity. This design was expected to allow us to directly compare the results to the existing evidence concerning individual memory (i.e. Bardach \& Park, 1996). Participant pairs discussed the characteristics of a stimulus person possessing SC and SI characteristics. To half of the participant pairs, the target was introduced as a member of the in-group. For the other half, she was described as an outgroup member. We tested the hypothesis that conversations about the out-group target would be more concentrated on SI than on SCinformation. On the other hand, we predicted that this predominance of SI utterances would be attenuated when the target was an in-group member because stereotypic expectations are likely to be weaker for the in-group than for the out-group (cf. Harasty, 1997).

\section{Method}

Participants and design Forty-four pairs of female undergraduates attending a large university (X University) in Western Japan volunteered to participate in the study. The members of each dyad were acquainted with each other when they came to the experiment. The pairs were randomly assigned to experimental conditions according to a 2 (group membership of the target: in-group vs. out-group) $\times 2$ (versions of the stimulus material) between-participant design.

Experimental materials An ostensive interview of a female undergraduate student was taperecorded and presented as the experimental stimulus. The responses by the interviewee alluded to some of her characteristics representing the stereotypes of X University and Y College. The two schools are located in the same city and are associated with roughly opposite stereotypes, with students of X University being perceived as academically oriented, relatively reserved, and typically coming from the middle class, whereas those at Y College are viewed as fashion-conscious, outgoing, and having highly affluent family backgrounds. To ascertain these stereotypic expectations, we conducted a pilot study in which we first generated a total of 89 behavioral descriptions that were thought to be stereotypic of each school. We then asked students enrolled at X University to rate the extent to which each sentence would stereotypically apply to students of X University, and 
separately to those of Y College on a 5-point scale ( $1=$ 'not at all', $5=$ 'a great extent'). Items that received the highest ratings for each school were selected for the use in the main experiment $(M \mathrm{~s}>$ 3.67 for X University, and $>3.83$ for Y College). The mean ratings of these items were all significantly different from the scale midpoint (i.e. $3.0 ; t s(11)$ $>2.60, p \mathrm{~s}<.03$ for $\mathrm{X}, t \mathrm{~s}(11)>3.08, p \mathrm{~s}<.01$ for $\mathrm{Y}$.) The final set of stimulus information is listed in the appendix.

In order to increase the generalizability of the stimulus and to avoid the salience effect of a particular domain of behavior, we generated two versions of the interviews (see appendix). That is, from a statement by the stimulus person in Version 1, 'I made a budget trip to Europe and stayed at cheap hotels' mentioned in the participants' conversation, we cannot ascertain whether this behavior was recalled because (as we intended) it was an 'X University SC', or because any episode involving overseas travel was particularly memorable. To cancel out such potential confounds between SC and domain salience, a behavior in the same domain but SI for X University (i.e. SC for Y College) was presented in Version 2 (i.e. 'I made an expensive trip to Europe'). As seen in the appendix, the two versions included such mirror-image stereotypes of these schools, with the number of total SC and SI items held constant.

Procedure A female undergraduate experimenter explained to the participants that the purpose of the study was to "examine how people form impressions of others based on visual and auditory information'. Participants first listened to a tape-recorded interview of the stimulus person which lasted for approximately five minutes. The experimenter mentioned the school affiliation of the stimulus person when she started to play the tape, thus assigning the participants randomly to the in-group or the out-group target condition. Photographs of the stimulus person were also presented. Next, participants were given a problem-solving task about the target. The task consisted of four questions that appeared to be related to the interview (e.g. 'Whether she prefers a Japanesestyle or Western-style breakfast'). Participants had to infer the true answer from the interview and were encouraged to verbalize whatever came to their minds in making their decisions. Because all questions were in a forced-choice format, each participant pair needed to reach consensus. The experimenter turned on the recorder and then excused herself from the laboratory room. Participants were allowed 10 minutes for discussion.

After coming back to the laboratory, the experimenter asked each participant to complete a questionnaire individually. First, participants were asked to rate the stimulus person, on a 7-point scale, with regard to five trait pairs that were relevant to the school stereotypes: 'poor wealthy', 'flashy - plain', 'introvert - extrovert', 'popular among men - not popular', 'fashionconscious - not conscious'. Scores from these five items were averaged for each participant because of the high reliability (Cronbach's $\alpha=.92$ ). Next, participants rated the target group as a whole regarding the same trait pairs $(\alpha=.91)$. After completing these tasks, participants were debriefed, thanked for their participation, and dismissed.

\section{Results}

Trait judgments Before analyzing the conversation data, we examined whether the stimulus information and the college stereotypes were operating on the present experimental setting in the expected manner. Specifically, we analyzed trait ratings of the stimulus person and the group as a whole which were made after the conversations. Because of the potential interdependency of the data within each pair, we treated dyads as the unit of analysis by averaging their scores.

We first attempted to ascertain that the impressions of the stimulus person did not differ depending on her group identity. Trait ratings of the target individual, with higher scores representing an impression of the 'feminine and out-going Y College type' (i.e. wealthy, flashy, extrovert, popular among men, and fashionconscious), were submitted to a 2 (group identity of the target person; in-group vs. out-group) $\times 2$ (version of the stimulus tape) analysis of variance (ANOVA) with both independent variables as 
between-participant. The ANOVA revealed a significant and unexpected main effect for the version $\left(F(1,40)=57.38, p<.0001, \eta^{2}=.589\right)$. Despite the careful piloting to develop equivalent stimulus characteristics, the person depicted in Version 2 was perceived to be more typical of Y College $(M=5.80)$ than the one in Version 1 $(M=3.98)$. Apparently, certain characteristics of the stimulus person that were stereotypic of $Y$ College dominated the overall impression of the person because the mean rating for this version significantly deviated from the midpoint of the scale (i.e. 4.0) $(t(21)=1.99, p<.001)$. (Note that this difference was observed regardless of the stimulus person's group identity, i.e. in- vs. out-group.) In contrast to Version 2, ratings of the person in Version 1 suggested that the participants formed an impression that we intended. Consistent with the evenly distributed SC and SI information in the stimulus, the mean rating was not significantly different from the midpoint $(t(21)<1, n s)$. The possibility that Version 2 may have been biased should be taken into consideration in the analysis of conversation contents, which we will report in the following section.

We next analyzed the ratings for the student body of the target school as a whole. The main effect for target identity was the only significant effect $\left(F(1,40)=125.72, p<.0001, \eta^{2}=.761\right)$. Consistent with the expected college stereotypes, students of the out-group (i.e. Y College) were rated to be the more affluent, female college student type $(M=5.72)$ than the in-group (i.e. $M=3.85)$. Hence, the school stereotypes seemed to have been introduced into our experimental setting successfully.

Contents of conversation From the transcripts of the conversations, we identified utterances that appeared to be relevant to the SC and SI information included in the original stimulus. Two coders blind to the experimental conditions went through the transcription of the conversations after receiving careful training. They identified phrases and sentences in the conversations that contained sufficient information to be defined as a reproduction of a stimulus item listed in the appendix. The intercoder agreement in this identification task was 97\% $($ Kappa $=.96)$ and disagreements were resolved by discussion. We then analyzed the reproduction data focusing on two main indices. First, we counted the number of SC and SI stimulus items reproduced by each dyad. Second, we measured the length of time that each item was mentioned.

A $2 \times 2 \times 2$ mixed model ANOVA was conducted for the number of utterances, with the SC of utterances (SC vs. SI) included as a withinparticipant factor. An unexpected effect of the stimulus version was indicated by a significant three-way interaction between the target's group identity, version, and stereotype-consistency $(F(1$, $\left.40)=58.13, p<.0001, \eta^{2}=.592\right)$. As Figure $2 \mathrm{a}$ illustrates, conversations based on Version 1 resulted in a greater number of SI than SC utterances concerning the out-group target $\left(F(1,40)=19.45, p<.001, \eta^{2}=.327\right)$. In contrast, conversations about the in-group member were more concentrated on SC than SI characteristics $\left(F(1,40)=10.38, p<.01, \eta^{2}=.206\right)$. Note that this was the stimulus scenario that produced well-balanced trait ratings on the target person, reflecting the evenly distributed SC and SI information.

On the other hand, Version 2, which was rated as more out-group-SC, produced a completely different pattern. Conversations about the out-group were more biased toward SC $(M=3.00)$ rather than SI information $(M=$ 1.73) $\left(F(1,40)=15.87, p<.001, \eta^{2}=.284\right)$. As for the in-group target, a greater number of utterances were made for SC $(M=1.18)$ than for SI characteristics $(M=2.64)(F(1,40)=$ $\left.16.77, p<.001, \eta^{2}=.295\right)$. The stimulus scenario which was biased toward the out-group stereotype has hence resulted in a reversed pattern of results.

Duration of utterances We next analyzed the overall duration of SC and SI utterances. ${ }^{2}$ Because the raw duration data showed a skewed distribution, log-transformed scores were submitted to a $2 \times 2 \times 2$ ANOVA. The interaction between group identity, version, and $\mathrm{SC}$ was again significant $\left(F(1,40)=56.98, p<.0001, \eta^{2}=.588\right)$. Figure $2 \mathrm{~b}$ illustrates the means from Version 1. When the participants listened to this version 


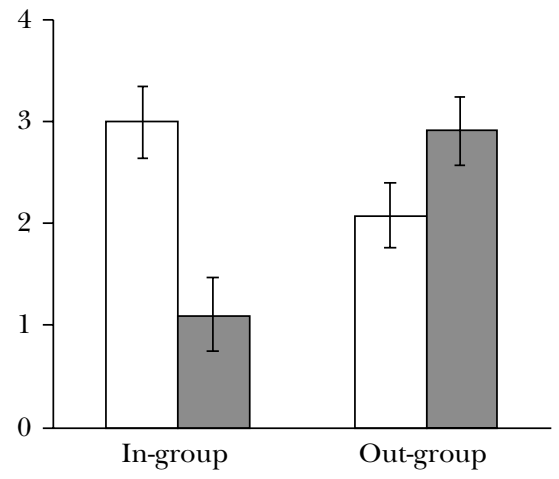

$\square$ Consistent $\square$ Inconsistent

(a) Number of utterances

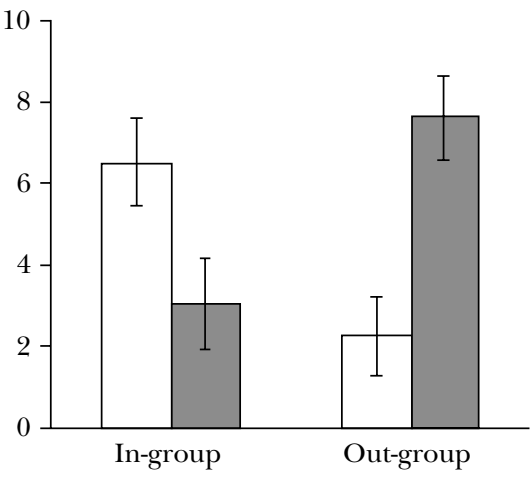

$\square$ Consistent $\square$ Inconsistent

(b) Duration of utterances (sec.)

Figure 2. Mean numbers and duration of SC and SI utterances: Study 1.

Note: Results from Version 1 alone.

describing the out-group member, significantly longer comments were made for SI than for SC characteristics $(F(1,40)=22.75, p<.001$, $\left.\eta^{2}=.363\right)$, while a nonsignificant, reversed pattern was found for the in-group target $(F(1$, $\left.40)=3.14, p<.09, \eta^{2}=.073\right)$. In contrast, Version 2 produced an opposite effect in that utterances for the out-group target were longer for SC $(M=9.12)$ than for SI $(M=3.80)(F(1$, $\left.40)=15.89, p<.001, \eta^{2}=.284\right)$, while utterances for the in-group were longer for SI $(M=5.97)$ than for SC $(M=1.59)$ characteristics $(F(1,40)=$ $\left.19.98, p<.001, \eta^{2}=.333\right)$. Hence, the potential problem with Version 2 mentioned earlier seems to have enhanced the reversal of the results across the different versions.

Correlation analyses To conduct correlational analyses, we computed the stereotypicality score of utterances by subtracting the number of SI comments from that of SC comments made by each individual. Likewise, the SC minus SI scores were computed for utterance durations as well. Following the method formulated by Gonzalez and Griffin (1997), the self-correlations (i.e. the association between the stereotypicality of utterances and ratings made by the same individual; $r_{\mathrm{ab}}$ in Figure 1) and cross-correlations (i.e. the association between one participant's utterances and ratings made by the conversation partner; $r_{\mathrm{ab}}$ ) were analyzed. ${ }^{3}$ The standardized correlation indices ( $Z$ scores) are shown in Table 1 (see also Griffin \& Gonzalez, 1995).

Here too, the two versions of stimulus interviews resulted in highly differentiated patterns. The results from Version 2, which are presumed to be biased toward the out-group (Y College) stereotype, were more straightforward. That is, regardless of the target group identity, the stereotypicality of the conversations (i.e. utterance counts as well as utterance duration) and that of impression ratings were significantly and positively correlated. This was the case for both self-correlations and cross-correlations. Remember that conversations based on this version were more concentrated on Y College SC information (i.e. in-group SI and outgroup SC). Presumably, the stimulus interview that was leaning toward the Y College stereotypes resulted both in conversations and subsequent ratings. On the other hand, Version 1 of the stimulus resulted in a highly contrasting pattern. For the rating of the target individual, the correlations between the conversation and the ratings were nearly null. However, the correlations between conversation and the group ratings were consistently and significantly negative.

It is worth noting that whenever there was a correlation, a cross-correlation accompanied the self-correlation. That is, the contents of 
Table 1. Standardized $(Z)$ scores of self- and cross-correlations between utterances and ratings: Study 1

\begin{tabular}{|c|c|c|c|c|c|}
\hline \multirow[b]{2}{*}{ Rating target } & \multirow[b]{2}{*}{$\begin{array}{l}\text { Stimulus } \\
\text { (n) }\end{array}$} & \multicolumn{2}{|c|}{ Utterance count } & \multicolumn{2}{|c|}{ Utterance duration } \\
\hline & & $\begin{array}{l}\text { Self } \\
\left(r_{\mathrm{ab}}\right) \\
\end{array}$ & $\begin{array}{c}\text { Cross } \\
\left(r_{\mathrm{ab}}\right)\end{array}$ & $\begin{array}{l}\text { Self } \\
\left(r_{\mathrm{ab}}\right)\end{array}$ & $\begin{array}{c}\text { Cross } \\
\left(r_{\mathrm{ab}}\right)\end{array}$ \\
\hline \multicolumn{6}{|l|}{ Individual } \\
\hline Version 1 & $(44)$ & .12 & 1.14 & -.40 & $1.98 *$ \\
\hline Version 2 & (44) & $3.37 * *$ & $4.33 * * *$ & $3.65^{* *}$ & $4.96 * * *$ \\
\hline Overall & (88) & $3.47 * *$ & $4.59 * * *$ & $3.43^{* *}$ & $5.39 * * *$ \\
\hline \multicolumn{6}{|l|}{ Group } \\
\hline Version 1 & $(44)$ & $-4.61 * * *$ & $-4.15 * * *$ & $-4.62 * * *$ & $-4.15 * * *$ \\
\hline Version 2 & $(44)$ & $2.83 * *$ & $4.18 * * *$ & $4.20 * * *$ & $3.29 * *$ \\
\hline Overall & $(88)$ & -1.40 & -.29 & $1.91 \dagger$ & 1.52 \\
\hline
\end{tabular}

$\dagger p<.10 ; * * p<.01 ; * * * p<.001$, two-tailed.

conversations were associated not only with the participant's own ratings (i.e. $r_{\mathrm{ab}}$ in Figure 1) but also with the partner's ratings $\left(r_{\mathrm{ab}},\right)$, after statistically controlling for dyadic interdependence. Restating from the reverse angle, an individual's ratings were correlated with the partner's utterances $\left(r_{\mathrm{a}^{\prime} \mathrm{b}}\right)$ as well as with her own $\left(r_{\mathrm{ab}}\right)$.

\section{Discussion}

The results of our first experiment revealed that stereotypic expectations of the in-group and the out-group influenced the contents of dyadic conversations. The effects varied, however, depending on the stimulus version. It should be first noted that Version 1 as the stimulus material produced the predicted pattern of results when the conversation target was an out-group member. Participants who heard this interview tape discussed more SI than SC characteristics of the target person and spent a longer time for SI comments. The rationale for our prediction of the predominance of SI conversations for the out-group was the assumption that SI information should be more surprising and thus elicit more elaborate processing. Indirect evidence for this assumption can be seen in the correlational data. The negative correlations between the utterance stereotypicality and group ratings suggest that participants who held stronger stereotypic expectations were more likely to make comments on SI characteristics. Or the correlation may indicate an opposite direction of effect in that those who talked more of SI characteristics might have subsequently rated the group (but not that particular individual) in a more stereotypic manner, thereby bolstering the group stereotypes and possibly 'subtyping' the given stimulus individual (see Hewstone, 1994; Weber \& Crocker, 1983). Indeed, further examination of the correlations revealed that the negative correlations were mainly found in the out-group target condition $(\mathrm{Zs}=-1.68$ for both self- and cross-correlations, both $p \mathrm{~s}<.10)$. Considering the small sample size $(n=20)$, these correlations are not negligible, at least compared to those found in the in-group target condition $(Z s=-0.11$ and 0.76 , respectively).

When the target depicted in Version 1 was presented as an in-group member, participants spent more time talking about her SC than SI characteristics. We predicted that the predominance of SI utterances would be attenuated in the in-group condition but did not anticipate that the effect could be even reversed. However, it may not be surprising to observe a predominance of SC utterances over SI in conversations. As mentioned in the introduction, this tendency has been repeatedly demonstrated in previous studies, especially when there is no necessity of reaching a correct answer (e.g. Kashima, 2000; Ruscher \& Duval, 1998). Likewise, the in-group target condition in the present study might have mitigated the perceived importance of the problem-solving task. That is, it is likely that the participants expected that they possessed 
relatively rich information about the in-group target and therefore presumed that they had already shared knowledge within the dyad. This presumption might have induced a more schema-based rather than piecemeal information processing about the target person (i.e. greater attention to SC than SI information).

Another unexpected result was that Version 2 of the stimulus scenario produced a pattern that was opposite to the conversations based on Version 1. When the target was presented as an out-group member, participants commented on a greater number of SC characteristics than SI characteristics and also spent a longer time talking about the former characteristics. Here too, a reversed pattern was found for the ingroup target. One possible explanation for this version effect is that particular depictions of the stimulus individual drew participants' special attention, especially when Version 2 was presented. Remember that the mean trait ratings of the stimulus person were clearly deviated from the midpoint of the scale toward the Y College stereotypes. This was the case regardless of the target's group identity. Thus, it is likely that particular episodes that were SC for Y College stereotypes in this version were especially distinct for some unknown reasons, and resulted in the conversations that were disproportionately concentrated on these Y-SC and X-SI characteristics. This interpretation was further corroborated by the correlational analyses. The stereotypicality in utterances showed consistently high positive correlations with the ratings of the target individual as well as the group. Hence, conversations as well as impression ratings based on this version of stimulus seems to have concentrated on Y College SC characteristics.

One may wonder, then, why this interpretation holds only for Version 2, but not for Version 1. That is, the patterns found in Figures 2a and $2 \mathrm{~b}$ may also be a mere result of certain salient characteristics in Version 1 that were SC for $\mathrm{X}$ University. We argue that this is unlikely to be a valid interpretation because the mean trait rating for this Version 1 was neutral. Also, the correlation between conversations and target ratings was generally low, suggesting that even when there was an especially salient characteristic for a particular participant, that information did not necessarily reflect on the conversation and the rating accordingly. Despite such indirect evidence that goes against the alternative interpretation on results from Version 1, the present design does not allow us to entirely rule out the potential confound. To solve this problem, we conducted a second experiment in which we recruited participants from Y College. If the above alternative explanation holds, then the conversations should be now relatively more dominated by 'out-group SC' and 'in-group SI' characteristics (i.e. X University SC in both cases), which is a reversed pattern compared to Study 1 (Version 1). Instead, if our interpretation of the results was valid, then the same target's identity by consistency interaction should be observed with Y College being the in-group and $\mathrm{X}$ University being the out-group.

On the basis of the foregoing interpretations, we tentatively conclude that when a target individual with a balanced set of SC and SI characteristics is presented, dyads talked more about SI characteristics in the out-group. In contrast, they talked more about in-group SC characteristics. These results are consistent with our hypothesis that SI information calls for more elaborate processing at the dyadic level, just as observed in intraindividual level of processes (Bardach \& Park, 1996). We attempted to replicate this result in Study 2.

\section{Study 2}

We conducted this second study with two main purposes. First, as already explained above, we collected the data at Y College in order to rule out the alternative explanations for the findings from Study 1, especially the one based on Version 1 of the stimulus. Second, we revised Version 2, attempting to introduce a more balanced stimulus person with regard to the school stereotypes.

\section{Method}

Participants and design Thirty-one pairs of female students of Y College participated in the study. The members of each dyad had already 
been acquainted with each other when they came to the experiment. The experimental design was again a 2 (group membership of the target: in-group vs. out-group) $\times 2$ (versions of the stimulus material) between-participant.

Experimental material and procedure In order to modify Version 2 of the stimulus interview into a more balanced set of descriptions, we deleted some extreme expressions from the items that were SC for Y College (see appendix). In addition, we allowed the participant pairs 5 minutes (instead of 10 minutes in Study 1) for their conversations. This was because the effects observed in Study 1 mainly took place during the first half of the conversations. The remainder of the experimental procedures was identical to Study 1, including the instruction for the dyadic conversations and the traits used for individual (Cronbach's $\alpha=.81$ ) and group ratings $(\alpha=.91)$.

\section{Results and discussion}

As in Study 1, the unit of analyses was the dyads of participants rather than individuals.

Trait judgments A $2 \times 2$ ANOVA was conducted for the averaged ratings of the target individual. The main effect for version was still significant $\left(F(1,27)=5.88, p<.05, \eta^{2}=.179\right)$, showing that the mean rating of the person in Version 2 $(M=4.93)$ remained more stereotypic of $\mathrm{Y}$ College compared to Version $1(M=4.25)$. However, as we intended, the mean rating of Version 2 was markedly moderated toward the scale midpoint, particularly compared to the mean observed in Study 1 (i.e. $M=5.80$ ). Turning to the averaged rating scores of the target group, an ANOVA revealed that the main effect for target was the only significant effect $(F(1,27)=155.02$, $\left.p<.0001, \eta^{2}=.852\right)$. As expected, the in-group was perceived to be more an 'out-going female college type' $(M=5.60)$ than the out-group $(M=3.43)$.

Contents of conversation The intercoder agreement in the classification of SC and SI reproductions was $95 \%(K a p p a=.93)$. A $2 \times 2 \times 2$ mixed-model ANOVA was conducted for the number of utterances, with target group identity, stimulus version, and stereotype consistency as independent variables. A moderate interaction was found between target identity and consistency $\left(F(1,27)=3.64, p<.07, \eta^{2}=\right.$ $.119)$. It should be noted that no interaction effect involving the version factor was significant $\left(F_{\mathrm{s}}<1.05\right.$, all $\left.n s, \eta^{2}<.038\right)$. The target identity $\times$ consistency interaction noted above showed exactly the same pattern in both versions. Figure $3 \mathrm{a}$ illustrates the results combining the two versions. The conversations concerning the out-group target included more SI utterances than SC $\left(F(1,27)=3.05, p<.09, \eta^{2}=.102\right)$, whereas there was no such difference for the in-group target condition $(F<1)$. The results were consistent with our prediction, and replicated the pattern observed in Study 1 with Version 1 as the stimulus scenario. At the same time, the results ruled out the alternative explanation for the results from Study 1. Because the ingroup versus out-group identity is now reversed between X University and Y College, the effect should be attributed to the combination of the target identity and stereotypicality rather than any specific characteristics of the stimulus.

Duration of utterances In the analysis of conversation time, we again submitted logtransformed scores to an ANOVA. The target $x$ consistency interaction now reached statistical significance $\left(F(1,27)=5.71, p<.05, \eta^{2}=.174\right)$. As illustrated in Figure 3b, participants spent more time discussing SI characteristics than SC of the out-group target $(F(1,27)=8.18$, $\left.p<.01, \eta^{2}=.233\right)$, whereas no difference between SC and SI was found for the in-group target $(F<1)$. Again, this result was consistent with our prediction. A caution is necessary, however, because the three-way interaction between target, consistency, and stimulus version also reached significance for this particular index of conversations $\left(F(1,27)=6.26, p<.05, \eta^{2}=.188\right)$. It was revealed that the pattern shown in Figure $3 \mathrm{~b}$ was particularly pronounced when Version 1 was presented, with conversations about the out-group target containing longer utterances for SI $(M=6.51)$ than for SC $(M=1.51)$ $\left(F(1,27)=11.72, p<.01, \eta^{2}=.303\right)$, and with 


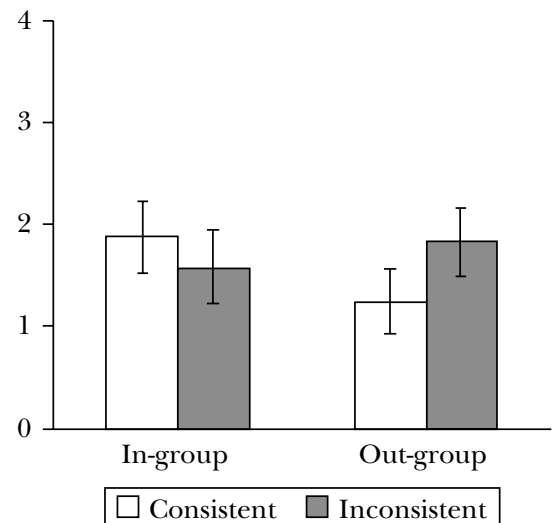

(a) Number of utterances

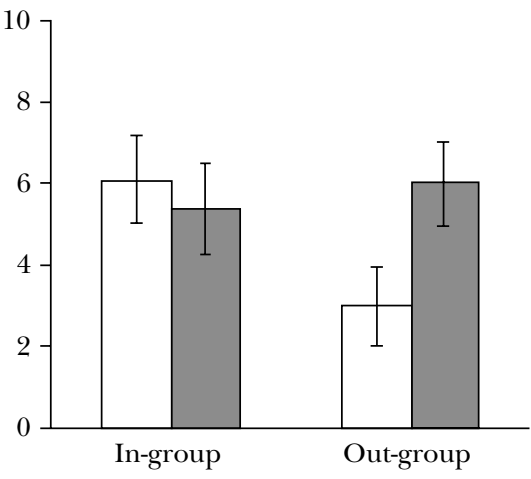

$\square$ Consistent $\square$ Inconsistent

(b) Duration of utterances (sec.)

Figure 3. Mean numbers and duration of SC and SI utterances: Study 2.

Note: Versions 1 and 2 combined.

a nonsignificant, reversed pattern for the in-group target $\left(M_{\mathrm{SC}}=6.23, M_{\mathrm{SI}}=2.74\right) \quad(F(1$, $27)=1.70, n s)$. However, these effects were visibly attenuated when Version 2 was presented, with no significant difference between the SC and SI utterances $(F<1)$ either for the out-group $\left(M_{\mathrm{SC}}=4.50, M_{\mathrm{SI}}=5.53\right)$ or for the in-group target $\left(M_{\mathrm{SC}}=5.76, M_{\mathrm{SI}}=8.30\right) .{ }^{4}$

In sum, the conversational data generally replicated the pattern that we found in Study 1 under the use of Version 1. (The effect of the stimulus versions was relatively moderated in Study 2.) The results confirmed that the observed effects were produced by the interaction between the target's group identity and the SC of the given information rather than as an artifact of some specifically salient topics in the stimulus.

Correlation analyses Unlike in Study 1 , the selfand cross-correlations did not show clear differentiation between the two stimulus versions, possibly due to smaller sample sizes (see Table 2). The stereotypicality of the conversation content was generally correlated positively with both the target impressions and the group impressions, particularly for the utterance count measure. Hence, the correlational results failed to replicate the ones from Study 1. It should be noted, however, that cross-correlations typically accompanied self-correlations. This means that whenever conversations were associated with the participant's own impression judgments, the association likely involved the participant's own utterances and judgments as well as the dyadic partner's. These results are consistent with our claim that stereotypes can be conceptualized as group representations that are formed both at the individual level and at the collectively shared level.

\section{General discussion}

The present study investigated stereotypes at a collective level by means of a behavioral index-dyadic conversations. This was among the first attempts to analyze such conversations in a single design with experimentally controlled $\mathrm{SC}$ and SI information and with in-group versus out-group targets. Separate studies in the past addressed similar issues, but each attempt fell short of a complete design. For example, Ruscher and her colleagues (e.g. Ruscher \& Hammer, 1994; Ruscher et al. 1996) used controlled stimuli and inspired a number of aspects of the present study, but they did not examine the effect of target identity. Harasty (1997) investigated the target effect, but the utterances examined in her study were based on participants' spontaneous statements and the experimenter did not have any control over the content. Another unique 
Table 2. Standardized $(Z)$ scores of self- and cross-correlations between utterances and ratings: Study 2

\begin{tabular}{|c|c|c|c|c|c|}
\hline \multirow[b]{2}{*}{ Rating target } & \multirow[b]{2}{*}{$\begin{array}{l}\text { Stimulus } \\
(\mathrm{n})\end{array}$} & \multicolumn{2}{|c|}{ Utterance count } & \multicolumn{2}{|c|}{ Utterance duration } \\
\hline & & $\begin{array}{l}\text { Self } \\
\left(r_{\mathrm{ab}}\right)\end{array}$ & $\begin{array}{c}\text { Cross } \\
\left(r_{\mathrm{ab}}\right)\end{array}$ & $\begin{array}{l}\text { Self } \\
\left(r_{\mathrm{ab}}\right)\end{array}$ & $\begin{array}{c}\text { Cross } \\
\left(r_{\mathrm{ab}}\right)\end{array}$ \\
\hline \multicolumn{6}{|l|}{ Individual } \\
\hline Version 1 & $(30)$ & $1.95^{\dagger}$ & $2.83 * *$ & 1.22 & $3.60 * *$ \\
\hline Version 2 & (32) & 1.25 & $2.17 *$ & 1.50 & $2.50 *$ \\
\hline Overall & (62) & $3.14 * *$ & $2.65^{* *}$ & 1.78 & 1.45 \\
\hline \multicolumn{6}{|l|}{ Group } \\
\hline Version 1 & $(30)$ & $2.36^{*}$ & 1.34 & .79 & -1.41 \\
\hline Version 2 & (32) & .95 & .86 & .08 & -1.59 \\
\hline Overall & (62) & $2.17 *$ & $2.19 *$ & $1.94^{\dagger}$ & 1.52 \\
\hline
\end{tabular}

$\dagger p<.10 ; * p<.05 ; * * p<.01$, two-tailed.

characteristic of the present study was the task. The problem-solving task was expected to impose a high need for consensus and accuracy on the participant pairs.

Across the two studies, it was consistently found that conversations based on an out-group target included more comments about SI than SC characteristics whereas comments about an in-group member showed an effect in the opposite direction, at least when the stimulus contained a balanced combination of SC and $\mathrm{SI}$ information. One of the stimulus versions used in Study 1 (i.e. Version 2) produced a different pattern, but this could be attributed to certain peculiar characteristics of the depicted person. In Study 2, where the stimulus materials were revised into a more balanced combination of SC and SI characteristics, the pattern was found to be more consistent with the predicted direction (i.e. a greater number of SI than SC utterances for the out-group, but no such difference for the in-group target). These results parallel the findings from research on the memory of individual targets. Whether the target is an individual (e.g. Hastie \& Kummar, 1979; Rojahn \& Pettigrew, 1992) or a group of people (Bardach \& Park, 1996), information that violates a prior expectation may result in a higher recall rate. Our results demonstrated that a similar effect was reflected in conversations when a pair of individuals attempted to form an integrated and consensual impression of the target.
It should be noted that the concentration on SI utterances was observed only for the outgroup target. Apparently, those SI characteristics shown by an in-group member were less surprising (Bardach \& Park, 1996) because the in-group is normally acknowledged to comprise a wider variation in characteristics than outgroups (i.e. the out-group homogeneity effect; e.g. Park et al., 1991).

The present results may appear to be incongruent with findings from some of the existing studies on stereotype-related conversations. For instance, a series of studies by Ruscher and colleagues (see Ruscher, 1998) generally showed the predominance of SC utterances. However, simplistic comparisons may be misleading because the methodologies employed in these studies are different in a number of ways. As we discussed in our introduction, there were a number of qualifications for the effects found by Ruscher and colleagues. In fact, their study showed that the combination of needs for consensus and accuracy facilitated the discussion of SI characteristics (Ruscher et al., 1996, Experiment 2). In this sense, the present results are in line with their findings.

A different experimental paradigm employed by Kashima (2000) also demonstrated the impact of SC information. Using a method of chained communication called 'serial reproduction', he revealed that SI characteristics may draw attention in earlier stages of information transmission, 
but that SC information eventually overrides the effect in a longer time span (see also Lyons \& Kashima, 2003). It should be noted, however, that the serial reproduction method investigates a unidirectional message transmission. Participants in this paradigm may well be aware that they will not receive feedback from the recipient of the transmitted message. Under such a circumstance, communicators may emphasize stereotypic information so that the recipient can easily form an impression of the target. In contrast, in dyadic conversations speakers expect immediate responses from their partner. Avoiding redundant communication about already-known aspects and discussing novel aspects may be more informative in this situation (Grice, 1975).

It is also important to note that the present task was designed to enhance a perceived need for accuracy. Participants were led to believe that there was a veridical 'correct' answer that was given by the target individual. It is well documented that perceivers under an accuracy goal pay greater attention to SI information (e.g. Fiske \& Neuberg, 1990; Ruscher \& Fiske, 1990). The same type of effect may have taken place in the present study at a collective level, drawing the attention of the dyads to SI characteristics, especially for the out-group target.

Taken together, the present results appear to complement, rather than contradict, the existing literature by specifying the contexts in which collective information processing is directed toward unexpected information. That is, conversations were drawn to SI information when the pairs were under a strong need to reach consensus for an accurate conclusion (i.e. the problem-solving task), with stimulus information containing evenly distributed SC and SI information. The effect was observed particularly in conversations about an outgroup target.

As a limitation of the present study, it may be pointed out that our prediction was supported mainly when the stimulus portrayed an individual with a balanced combination of $\mathrm{SC}$ and SI characteristics (i.e. Version 1). Version 2 used in Study 1 was found to be biased toward the out-group stereotype, and resulted in a reversed pattern. We attempted to revise the stimulus into a neutral characteristic in Study 2, but apparently failed to construct a completely balanced figure, according to the impression ratings. Consequently, the measure of utterance length still did not replicate the result obtained from Version 1, even though in terms of the utterance count measure we did successfully remove significant interaction effects involving the scenario factor. Further examinations are needed to resolve the ambiguity concerning specific information given in different stimulus materials in order to determine the generalizibility of the present findings.

Another limitation may lie in the selection of concrete groups and stereotypes. In the present study, we intended to choose two groups that involved nearly a mirror image of school stereotypes. However, the actual images of the two schools may not have been exactly the opposite. Also, the stereotype of Y College (i.e. fun-loving female college students) appeared to have been stronger than that of $\mathrm{X}$ University. These asymmetries in school stereotypes seem to have caused the difficulty in developing equivalent stimulus versions for counterbalancing and in replicating every aspect of the results across the two studies. For instance, the correlational data from Study 1 appeared to provide indirect support for our assumption that SI characteristics of an out-group member elicit more elaborate processing. That is, consistently negative correlations were found between the stereotypicality of utterances and stereotypic ratings of the target group, especially in the out-group condition. However, this was the case only for Version 1, and more important, the correlational pattern was not replicated in Study 2. Further investigations, with careful selection of stimulus materials from a wider variety in groups and their stereotypes, are needed to obtain robust evidence for the present arguments.

Despite these potential problems, the present study demonstrated the importance of studying stereotypes as collectively shared group representations. Such importance may be easy to 
point out, but implementing empirical research proves to be a difficult task. The present study has demonstrated that incorporating diverse perspectives and different methodologies can provide a solution to such difficulties. Note that the present study employed a number of conceptual tools that were originally developed by previous investigations on the individual level of cognitive processes. As the present study has demonstrated, ideas concerning individual level processes, such as integration and elaboration of SI versus SC information, can certainly guide the designing of empirical research on collective level phenomena. We also showed that analyzing communication processes among dyads can provide a fruitful way to study collective level concepts. Of particular importance was the finding that the conversations may influence not only the participant's own judgments but also the partner's judgments (or own held stereotypes influencing the partner's utterances). Further elaboration of such methodologies that tap into the interrelatedness among people and among different processes will likely benefit this line of research substantially. Integrating these diverse views and analyses, the study of stereotypes and prejudices should acquire a wider perspective toward an understanding of truly 'social' characteristics of intergroup attitudes.

\section{Notes}

1. In their original formulation, Gonzalez and Griffin (1997) coined the terms 'overall correlation' and 'cross intraclass correlation', respectively, to refer to these. However, we decided to adopt the present terminology because we found it to be more straightforward. It is also important to note that the members of each pair are interchangeable and are both included in the computation of correlations. In other words, $r_{a b}$ and $r_{a^{\prime} b}$, are practically identical to each other, and so are $r_{a b}$, and $r_{a b}$ (see Griffin \& Gonzalez, 1995; Gonzalez \& Griffin, 1997).

2. For the number of utterances, we excluded repetitions from the analysis because our interest was mainly in revealing what aspects of the stimulus character were reflected in the conversation. In contrast, as for the time measurement, we included the repetitions in order to examine the overall representations of the target. However, including or excluding repetitions in each index led us to the same conclusions about the results.

3. In this particular analysis, ratings of the target individual and the group were both reversed only for the in-group target condition so that higher scores always represented stereotypic judgments about the respective target.

4. The only other significant effect was the main effect of version $(F(1,26)=5.51 p<.05$, $\left.\eta^{2}=.169\right)$. This indicated that participants spend a longer time discussing stereotyperelevant characteristics (including both $\mathrm{SC}$ and SI) on the basis of Version $2(M=6.02)$ than on Version $1(M=4.25)$. This effect provides no important information for the present purpose.

\section{Acknowledgements}

The present study was supported by a Grant-inAid for Scientific Research from the Japan Society for the Promotion of Science (15330134) and by the Center for Legal Dynamics of Advanced Market Society at Kobe University. We thank Ayako Kawai, Yuka Kiriki, Yuki Mori, Masayo Oh'i, Junko Oh'shita, Naoko Ozoe, Aya Takahashi, and Fumiko Yamamoto for their assistance in data collection and coding, and Christian Chan for his help in preparing this manuscript.

\section{References}

Bardach, L., \& Park, B. (1996). The effects of in-group/out-group status on memory for consistent and inconsistent behavior of an individual. Personality and Social Psychology Bulletin, 22, 169-178.

Dijksterhuis, A., \& van Knippenberg, A. (1995). Timing of schema-activation and memory: Inhibited access to inconsistent information. European Journal of Social Psychology, 25, 383-390.

Fiske, S. T., \& Taylor, S. E. (1991). Social cognition. New York: McGraw-Hill.

Fiske, S. T., \& Neuberg, S. L. (1990). A continuum of impression formation, from category-based to individuating processes: Influences of information and motivation on attention and interpretation. In M. P. Zanna (Ed.), Advances in experimental social psychology (Vol. 23, pp. 1-74). New York: Academic Press. 
Gardner, R. C. (1994). Stereotypes as consensual beliefs. In M. P. Zanna \& J. M. Olson (Eds.), The psychology of prejudice: The Ontario symposium (Vol. 7, pp. 1-31). Hillsdale, NJ: Erlbaum.

Gonzalez, R., \& Griffin, D. (1997). On the statistics of interdependence: Treating dyadic data with respect. In S. Duck (Ed), Handbook of personal relationships: Theory, research and interventions (2nd ed., pp. 271-302). Chichester, UK: Wiley.

Grice, H. P. (1975). Logic and conversation. In P. Cole \& J. L. Morgan (Eds.), Syntax and semantics: Vol. 3. Speech acts (pp. 41-58). New York: Academic Press.

Griffin, D., \& Gonzalez, R. (1995). Correlational analysis of dyad-level data in the exchangeable case. Psychological Bulletin, 118, 430-439.

Harasty, A. S. (1997). The interpersonal nature of social stereotypes: Differential discussion patterns about in-groups and out-groups. Personality and Social Psychology Bulletin, 23, 270-284.

Hardin, C. D., \& Higgins, E. T. (1996). Shared reality: How social verification makes the subjective objective. In R. M. Sorrentino \& E. T. Higgins (Eds.), Handbook of motivation and cognition: Vol 3. The interpersonal context (pp. 28-84). New York: Guilford.

Haslam, S. A., Oakes, P. J., McGarty, C., Turner, J. C., Reynolds, K. J., \& Eggins, R. A. (1996). Stereotyping and social influence: The mediation of stereotype applicability and sharedness by the views of in-group and out-group members. British Journal of Social Psychology, 35, 369-397.

Hastie, R., \& Kumar, P. A. (1979). Person memory: Personality traits as organizing principles in memory for behavior. Journal of Personality and Social Psychology, 37, 25-38.

Hewstone, M. (1994). Revision and change of stereotypic beliefs: In search of the elusive subtyping model. In W. Stroebe \& M. Hewstone (Eds.), European review of social psychology (Vol. 5, pp. 69-109). Chichester, UK: Wiley.

Higgins, E. T., \& Rholes, W. S. (1978). 'Saying is believing': Effects of message modification on memory and liking for the person described. Journal of Experimental Social Psychology, 14, 363-378.

Kashima, Y. (2000). Maintaining cultural stereotypes in the serial reproduction of narratives. Personality and Social Psychology Bulletin, 26, 594-604.
Klein, O., \& Snyder, M. (2003). Stereotypes and behavioral confirmation: From interpersonal to intergroup perspectives. In M. P. Zanna (Ed.), Advances in experimental social psychology (Vol. 35, pp. 153-234). San Diego, CA: Academic Press.

Linville, P. W., \& Jones, E. E. (1980). Polarized appraisals of out-group members. Journal of Personality and Social Psychology, 38, 689-703.

Linville, P. W., Salovey, P., \& Fischer, G. W. (1986). Stereotyping and perceived distributions of social characteristics: An application to ingroup-outgroup perception. In J. Dovidio \& S. L. Gaertner (Eds.), Prejudice, discrimination, and racism (pp. 165-208). New York: Academic Press.

Lyons, A., \& Kashima, Y. (2003). How are stereotypes maintained through communication? The influence of stereotype sharedness. Journal of Personality and Social Psychology, 85, 989-1005.

Maass, A. (1999). Linguistic intergroup bias: Stereotype perpetuation through language. In M. P. Zanna (Ed.), Advances in experimental social psychology (Vol. 31, pp. 79-121). San Diego, CA: Academic Press.

Oakes, P. J., Haslam, S. A., \& Turner, J. C. (1994). Stereotyping and social reality. Oxford, UK: Blackwell.

Park, B., \& Judd, C. M. (1990). Measures and models of perceived group variability. Journal of Personality and Social Psychology, 52, 173-191.

Park, B., Judd, C. M., \& Ryan, C. S. (1991). Social categorization and the representation of variability information. In W. Stroebe \& M. Hewstone (Eds), European review of social psychology (Vol. 2, pp. 211-245). Chichester, UK: Wiley.

Rojahn, K., \& Pettigrew, T. F. (1992). Memory for schema-relevant information: A meta-analytic resolution. British Journal of Social Psychology, 31, 81-109.

Ruscher, J. B. (1998). Prejudice and stereotyping in everyday communication. In M. P. Zanna (Ed.), Advances in experimental social psychology (Vol. 30, pp. 241-307). San Diego, CA: Academic Press.

Ruscher, J. B. (2001). Prejudiced communication: A social psychological perspective. New York: Guilford.

Ruscher, J. B., \& Duval, L. L. (1998). Multiple communicators with unique target information transmit less stereotypical impressions. Journal of Personality and Social Psychology, 74, 329-344. 
Ruscher, J. B., \& Fiske, S. T. (1990). Interpersonal competition can cause individuating processes. Journal of Personality and Social Psychology, 58, 832-843.

Ruscher, J. B., \& Hammer, E. D. (1994). Revising disrupted impressions through conversation. Journal of Personality and Social Psychology, 64, 530-541.

Ruscher, J. B., Hammer, E. Y., \& Hammer, E. D. (1996). Forming shared impressions through conversation: An adaptation of the Continuum Model. Personality and Social Psychology Bulletin, 22, 705-720.

Snyder, M., \& Uranowitz, S. W. (1978). Reconstructing the past: Some cognitive consequences of person perception. Journal of Personality and Social Psychology, 36, 941-950.

Srull, T. K., \& Wyer, R. S., Jr. (1989). Person memory and judgement. Psychological Review, 96, 58-83.

Stangor, C., \& McMillan, D. (1992). Memory for expectancy-congruent and expectancyincongruent information: A review of the social and social developmental literatures. Psychological Bulletin, 111, 42-61.

Stangor, C., \& Schaller, M. (1996). Stereotypes as individual and collective representations. In C. N. Macrae, C. Stangor, \& M. Hewstone (Eds.), Stereotypes and stereotyping (pp. 3-37). New York: Guilford.

Stasser, G., \& Stewart, D. (1992). Discovery of hidden profiles by decision-making groups: Solving a problem versus making a judgment. Journal of Personality and Social Psychology, 63, 426-434.

Stasser, G., \& Titus, W. (1985). Pooling of unshared information in group decision making: Biased information sampling during group discussion. Journal of Personality and Social Psychology, 48, 1467-1478.

Van Dijk, T. (1987). Communicating racism: Ethnic prejudice in thought and talk. London: Sage.

Weber, R., \& Crocker, J. (1983). Cognitive processes in the revision of stereotypic beliefs. Journal of Personality and Social Psychology, 45, 961-977.

Wigboldus, D. H. J., Semin, G. R., \& Spears, R. (2000). How do we communicate stereotypes? Linguistic bases and inferential consequences. Journal of Personality and Social Psychology, 78, 5-18.

Paper received 5 May 2006; revised version accepted 1 August 2006.

\section{Biographical notes}

MINORU KARASAWA is an associate professor of psychology at Nagoya University. He received his PhD from the University of California, Los Angeles. His main research interests are in stereotyping and intergroup relations. In particular, he has been studying collectively shared aspects of stereotypes by examining the influence of communicative contexts. He is also interested in more broad issues involving shared cognition, such as the role of culture and language in social cognition.

NOBUko Asai is a PhD student at the Graduate School of Humanities and Social Sciences of Kobe University. Her research interests are in stereotyping, psychological essentialism, and prejudices. In her dissertation research, she is examining causal attribution and coping strategies among victims of prejudices and discrimination.

YOSHIKO TANABE is a doctorate student at the Graduate School of Humanities and Social Sciences of Kobe University. She has been studying the effect of group stereotypes on language and communication. Her research interest also extends to a developmental study of the mother-child relationship.

\section{Appendix}

\section{Stereotype-consistent and inconsistent items in the stimulus}

\section{Version 1}

\section{University-SC (Y College-SI)}

1. Member of a tennis club that is highly disciplined with a strict senior-junior status code.

2. Made a budget trip to Europe during the summer break and always stayed at cheap hotels.

3. Normally wear low-key outfits. Not interested in expensive brands.

4. In charge of housekeeping because both parents work full-time.

5. Having a hard time making boyfriends because easily get uptight when meeting men. 


\section{Y College-SC (X University-SI)}

1. Nearly flunked a computer programming class because could not learn well. Hate to see a computer.

2. Often go to parties to meet men.

3. Typically hang out on holidays, having teas during the day and going for drinks at night.

4. Failed to complete a part-time job as a home tutor because was not a good teacher.

5. Pointed out by friends to be overly easygoing and optimistic.

\section{Version 2}

\section{Y College-SC (X University-SI)}

1. Member of a tennis club. The main purpose of the club is to have fun (with cute guys). ${ }^{a}$

2. Made an expensive trip to Europe during the summer break (to stay at an expensive hotel with gourmet tours).
3. Normally wear (more) flashy outfits (than today).

4. Never do housekeeping because the mother is a full-time housewife (because the family employs a maid).

5. Dating a steady boyfriend, but do not hesitate to go out with (many) other male friends.

\section{University-SC (Y College-SI)}

1. Did well in a computer programming class, and wrote a program of a computer game.

2. Do not like to go to parties set up for meeting men.

3. Typically stay home on holidays to read and bake cookies.

4. Like to teach and work hard as a home tutor for a part-time job.

5. Described by friends as a stable and reliable person.

a The parts in parentheses were used in Study 1 but deleted or replaced in Study 2. 